\title{
JNK modulates FOXO3a for the expression of the mitochondrial death and mitophagy marker BNIP3 in pathological hypertrophy and in heart failure
}

\author{
AH Chaanine ${ }^{1}$, D Jeong ${ }^{1}$, L Liang ${ }^{1}$, ER Chemaly ${ }^{1}$, K Fish ${ }^{1}$, RE Gordon ${ }^{2}$ and RJ Hajjar ${ }^{\star, 1}$
}

Bcl-2 E1B 19-KDa interacting protein 3 (BNIP3) is a mitochondrial death and mitophagy marker, which is involved in inducing cardiac remodeling post myocardial infarction. In this study, we show that BNIP3 expression increases in stressed cardiomyocytes in vitro and in response to pressure overload in vivo, and that its transcription is directly related to JNK activity. BNIP3 expression gradually increased in the first weeks after pressure overload and peaked at the heart failure stage. Ultrastructurally, the mitochondrial area was inversely proportional to BNIP3 expression. Both JNK and AKT activities increased with pressure overload; however, JNK signaling dominated over AKT signaling for the activation of the transcription factor FOXO3a and for the transcription of its effector, BNIP3. 3-methyladenine attenuated JNK signaling and significantly decreased BNIP3 expression and reversed cardiac remodeling in heart failure. Ultrastructurally, the mitochondrial area was significantly increased in the 3-methyladenine group compared with placebo. Moreover, adenoviral gene delivery of dominant negative JNK in a rat model of pressure overload hypertrophy abolished the increase in BNIP3 expression in response to pressure overload. These results suggest that JNK signaling is a critical modulator of the transcription factor FOXO3a driving the expression of its effector, BNIP3, in heart failure and that JNK, through BNIP3, induces mitochondrial apoptosis and mitophagy.

Cell Death and Disease (2012) 3, e265; doi:10.1038/cddis.2012.5; published online 2 February 2012

Subject Category: Internal Medicine

Heart failure is a clinical syndrome characterized by the activation of the neurohormonal and renin angiotensin aldosterone system followed by remodeling of the left ventricle (LV) and alterations in the LV geometry. ${ }^{1}$ The integrity of the endoplasmic reticulum (ER) and the juxtaposed mitochondria is pivotal for the proper function of the cardiomyocyte. Ultrastructurally, these two organelles are located at very close proximity and crosstalk with each other via calcium signaling. ${ }^{2-6}$ In heart failure, both the ER and the mitochondria, and each on its own, execute death signals that take the form of programmed apoptotic and autophagic cell death. ${ }^{7}$ The decline in cardiac function of heart failure patients is in part due to the loss of the diseased cardiac myocytes in the form of necrotic, apoptotic and autophagic cell death. The $\mathrm{Bcl}-2$ family proteins serve as a critical death regulators that reside immediately upstream of the mitochondria. They consist of anti-apoptotic members such as Bcl-2 protein and pro-apoptotic members. The pro-apoptotic Bcl-2 members are subdivided into 'multidomain' and ' $\mathrm{Bcl}-2$ Homology ( $\mathrm{BH} 3)$ ' proteins. Multidomain pro-apoptotic proteins such as Bax and Bak display sequence conservation in the $\mathrm{BH}$ domains 1-3 and their expression is directly regulated by the anti-apoptotic $\mathrm{Bcl}-2$ protein. On the other hand, the $\mathrm{BH} 3-$ only members display sequence conservation only in the $\alpha$-helical $\mathrm{BH} 3$ region, which constitutes the critical death domain. ${ }^{8}$ Of the $\mathrm{BH} 3$ members, the $\mathrm{Bcl}-2 \mathrm{E} 1 \mathrm{~B} 19-\mathrm{KDa}$ interacting protein 3 (BNIP3) is unique in the sense that it induces mitochondrial apoptosis as well as mitochondrial autophagy (mitophagy). ${ }^{9}$ In the initial phase of apoptosis, BNIP3 inserts into the outer mitochondrial membrane with the $\mathrm{N}$ terminus oriented into the cytoplasm and the $\mathrm{C}$ terminus inside the mitochondria. It induces mitochondria-mediated apoptosis and fragmentation by driving mitochondrial permeability transition pore opening, cytochrome $\mathrm{C}$ release and the destruction of the mitochondrial cristae. $^{10,11}$ On the other hand, BNIP3 is an autophagy receptor that activates mitophagy in a non-canonical order leading to their sequestration and subsequent removal. ${ }^{9,12,13}$ What makes BNIP3 more interesting is that, unlike the other $\mathrm{Bcl}-2$ members, it is the effector of the transcription factor FOXO3a in post-mitotic skeletal muscles and cardiomyocytes. ${ }^{14}$ Many studies have suggested that BNIP3 expression increases under ischemic condition in cardiac myocytes and that cardiac remodeling is directly related to BNIP3 expression. ${ }^{15-19}$ In this study, we show that BNIP3 is expressed in response to cardiomyocyte stressors, such as phenylephrine (PE) or calcium, in vitro and to pressure overload in vivo.

\footnotetext{
${ }^{1}$ Cardiovascular Institute, Mount Sinai School of Medicine, New York, NY 10029, USA and ${ }^{2}$ Pathology Department, Mount Sinai School of Medicine, New York, NY 10029, USA

*Corresponding author: RJ Hajjar, Cardiology, Mount Sinai School of Medicine, One Gustave L. levy Place, Box 1030, New York, NY 10029, USA. Tel: + 1212241 4082; Fax: + 1212241 4080; E-mail: roger.hajjar@mssm.edu

Keywords: heart failure; JNK; FOXO3a; BNIP3; mitochondrial apoptosis; mitophagy

Abbreviations: ER, endoplasmic reticulum; JSR, junctional sarcoplasmic reticulum; IMFM, intermyofibrillar mitochondria; mPTP, mitochondrial permeability transition pore; BNIP3, Bcl-2 E1B 19-KDa interacting protein 3; PE, phenylephrine; 3 MA, 3-methyladenine; Ad-GFP, adenovirus green fluorescent protein; Ad-Null, adenovirus Null; Ad-DN-JNK, adenovirus dominant negative JNK; Ad-FOXO3a, adenovirus constitutively active FOXO3a; Ad-DN-FOXO3a, adenovirus dominant negative FOXO3a; Ad-BNIP3, adenovirus BNIP3; Ad-Sh BNIP3, adenovirus small hairpin BNIP3; Ad-eGFP-LC3, adenovirus enhanced green fluorescent -light chain 3 protein Received 12.12.11; revised 05.1.12; accepted 05.1.12; Edited by RA Knight
} 
Moreover, we show how the interplay between AKT and JNK signaling modulates $\mathrm{FOXO} 3 \mathrm{a}$ for the transcription of its effector BNIP3. Moreover, we show that 3-methyladenine (3 MA), by interfering with JNK signaling, modulates the expression of the mitochondrial death and mitophagy marker BNIP3 in vivo, and reverses cardiac remodeling in a rat model of pressure overload-induced heart failure. This signaling pathway was further validated via the adenoviral gene delivery of dominant negative JNK (Ad-DN-JNK) in a rat model of pressure overload hypertrophy $(\mathrm{POH})$.

\section{Results}

BNIP3 is the effector of FOXO3a and its expression is upregulated in PE-stressed cardiomyocytes. 3 MA inhibited the increase in BNIP3 expression in PE-stressed cardiac myocytes in vitro. BNIP3 expression is increased by two-fold $2 \mathrm{~h}$ after cardiomyocyte stress with $\mathrm{PE}$ or calcium in vitro. BNIP3 expression was also increased with the addition of chloroquine, a well-known autophagolysosome fusion inhibitor, in stressed cardiomyocytes, whereas $3 \mathrm{MA}$, an autophagy induction inhibitor, inhibited the increase in BNIP3 expression Figure 1a. The relative BNIP3 mRNA expression was significantly increased in PE-stressed cardiomyocytes for $2 \mathrm{~h}$ and its increase was significantly inhibited by $3 \mathrm{MA}$ Figure 1b. Autophagosomes, containing mitochondria with different stages of vacuolar degeneration, were observed in chloroquine, PE and PE plus chloroquine-stressed cardiomyocytes Figure 1c and Supplementary Figure 1. The number of autophagosomes increased with the addition of chloroquine and in PE-stressed cardiomyocytes for $2 \mathrm{~h}$ with the highest number of autophagosomes observed in the PE plus chloroquine-treated cardiomyocytes. $3 \mathrm{MA}$ inhibited the formation of autophagosomes in PE-stressed cardiomyocytes Figure 1d. This suggests that the increase in autophagosomes with PE treatment is due to the increase in autophagosomes formation rather than the consequence of a degradation removal problem. The overexpression of FOXO3a using an adenovirus containing constitutively active FOXO3a (Ad-FOXO3a) increased the expression of BNIP3 in cardiac myocytes in vitro compared with adenovirus green fluorescent protein (Ad-GFP) and dominant negative FOXO3a (Ad-DN-FOXO3a), respectively Figure 1e. Moreover, the overexpression of BNIP3 in eGFP-LC3 expressing cardiomyocytes by simultaneous infection with an adenovirus containing BNIP3 (Ad-BNIP3) and another with eGFP-LC3 (Ad-eGFP-LC3), robustly increased the number of autophagosomes compared with adenovirus Null (Ad-Null) and adenovirus Sh BNIP3 (Ad-Sh BNIP3)-infected cardiomyocytes, respectively Figure 1f. Western blot data shown in Supplementary Figure 2. Ultrastructurally, BNIP3 overexpression in cultured cardiac myocytes was associated with a marked increase in autophagosomes and robust decrease in mitochondrial area compared with Ad-Null and Ad-Sh BNIP3infected cardiac myocytes, respectively Figure $1 \mathrm{~g}$ and Supplementary Figure 3.

3 MA reversed cardiac remodeling and improved cardiac function in heart failure. $3 \mathrm{MA}$ treatment reversed cardiac remodeling in heart failure compared with the placebo group as shown in Figure $2 a$. Transthoracic echocardiography data are shown in the Supplementary Table 1. There was no difference in the heart weight versus the body weight in both the groups. There was no difference in LV septal (LVSd) and posterior wall (LVPWd) thickness between the 3 MA and the placebo groups. However, there were significant decreases in the LV end diastolic diameter (LVIDd), LV end diastolic volume (LVEDV), LV end systolic diameter (LVIDs) and LV end systolic volume (LVESV) in the $3 \mathrm{MA}$ group compared with the placebo group, respectively Figure $2 \mathrm{~b}$. This was accompanied by significant increases in LV fractional shortening and LV ejection fraction in the 3 MA group compared with the placebo group Figure 2c. Hemodynamic data are shown in the Supplementary Table 2. There was a trend in improved LV contractility and efficiency at baseline as determined by pressure-volume loop measurements in the 3 MA group compared with the placebo group, but with a statistically significant shift of V0 to the left in the $3 \mathrm{MA}$ group compared with placebo Figure 2d and Supplementary Table 2. The 3 MA group had significantly lower end diastolic pressure, tau and end-diatloic pressure-volume relationships (EDPVR) compared with the placebo group suggesting improved relaxation of the LV. Moreover, The 3 MA group significantly increased their LV contractility with $\beta$-adrenergic stimulation in response to escalating doses of dobutamine infusion compared with the placebo group Figure 2e.

3 MA significantly decreased the expression of FOXO3a effector, BNIP3 and MurF-1, and restored mitochondrial cristae in heart failure. The expression of FOXO3a effectors, the mitophagy marker BNIP3 and the atrophy marker MurF-1, are significantly upregulated in heart failure compared with age-matched controls. 3 MA significantly attenuated the expression of these markers in heart failure compared with the placebo group Figure 2f. Ultrastructurally, the placebo group had tortuous-looking myofibers with myofibrillar disarray and an increase in the autophagolysosomes. Also, there was dilatation of the junctional sarcoplasmic reticulum (JSR) and T-tubules, most of them being occupied with lamellar structures. The intermyofibrillar mitochondria (IMFM) were fragmented with severe loss in their area and hazy-looking cristae compared with agematched control. 3 MA treatment reversed the tortuousity of the myofibers and the myofibrillar disarray, but did not improve JSR dilatation. $3 \mathrm{MA}$ treatment significantly decreased IMFM fragmentation as measured by the decrease in the average area per mitochondrion and restored their cristae Figures $2 g$ and $h$. Figure $2 i$ show the correlation between BNIP3 expression and the average area per mitochondrion. The decrease in BNIP3 expression by 3 MA resulted in significant increase in the average area per mitochondrion.

Prolonged JNK activation overrides the inhibitory effect of AKT on FOXO3a and activates the latter for the expression of its effectors, BNIP 3 and MurF-1. JNK and AKT have opposite effects on FOXO3a. JNK activates FOXO3a whereas AKT inhibits its activity. Moreover, it has 
a

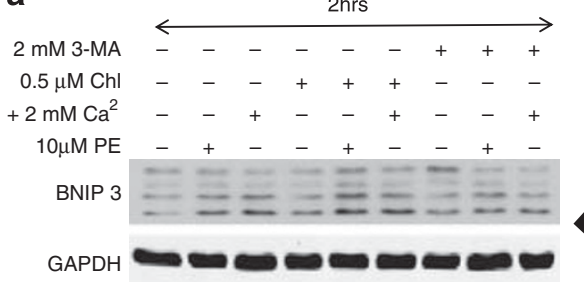

C
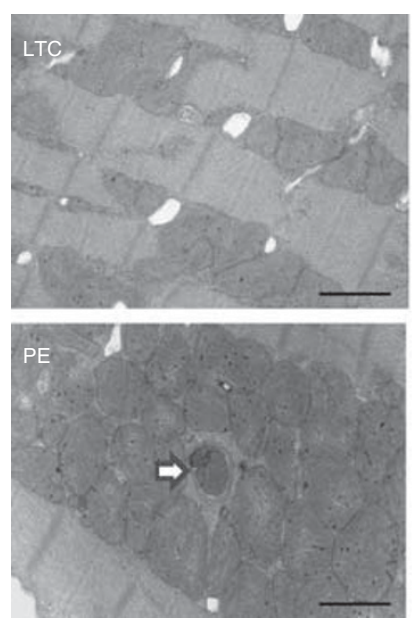

d

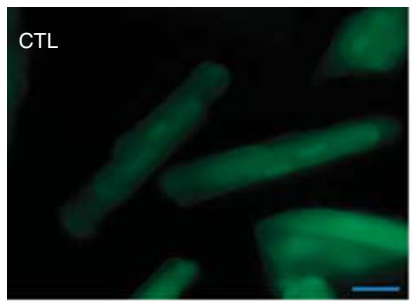

PE, 2hrs

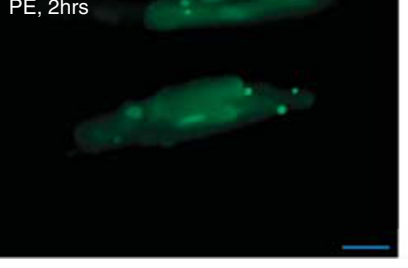

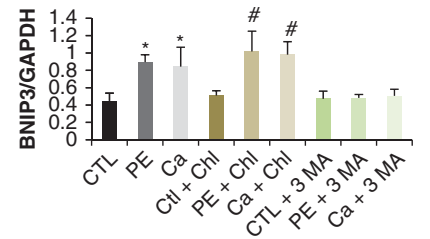
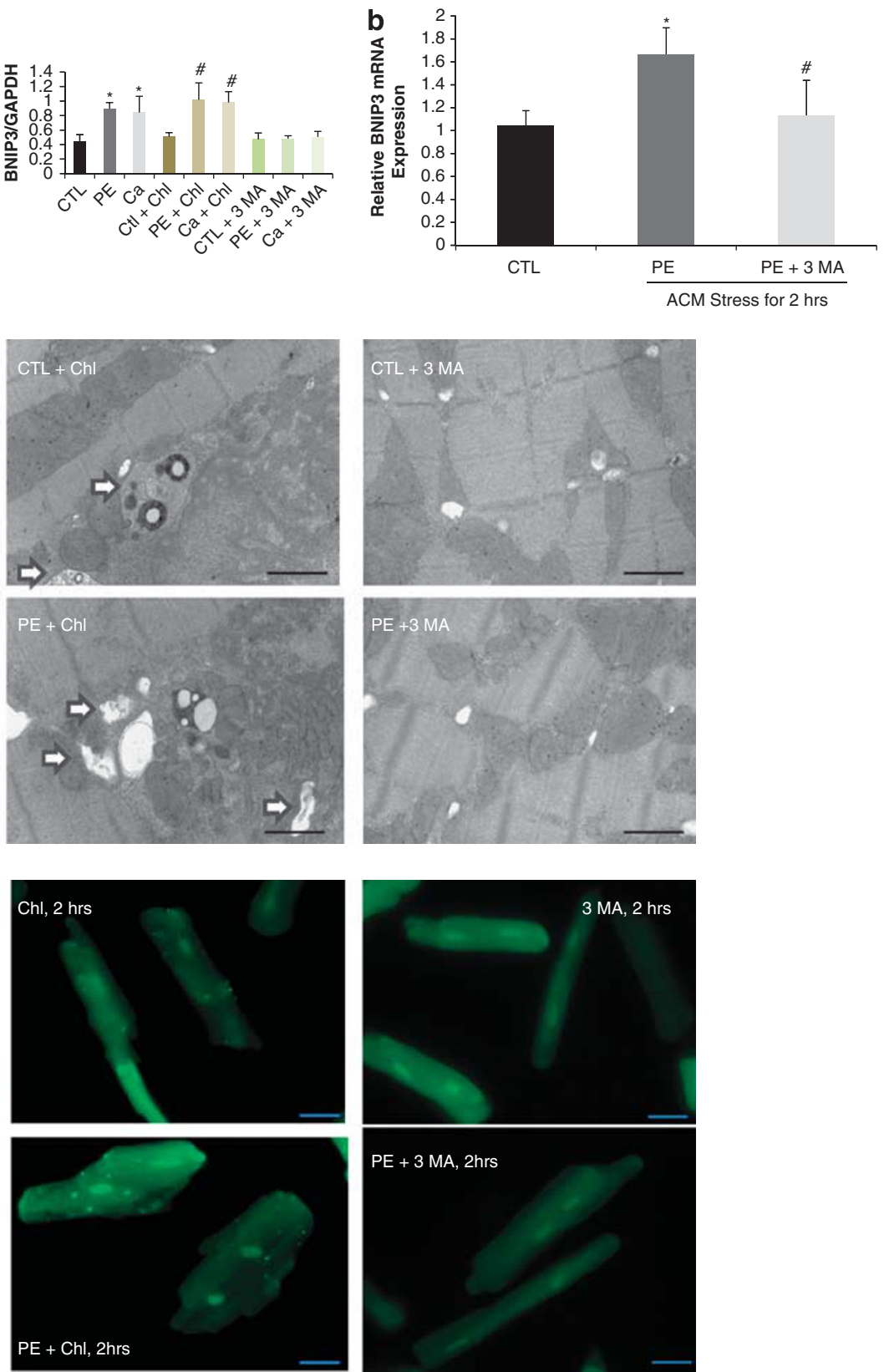

$\mathrm{PE}+3 \mathrm{MA}, 2 \mathrm{hrs}$

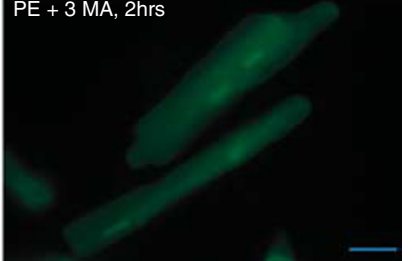

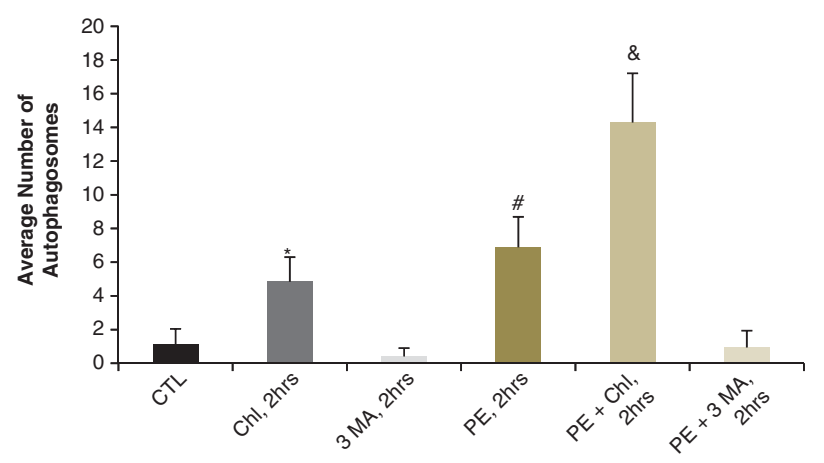

Figure 1 (Continued) 


\section{e}

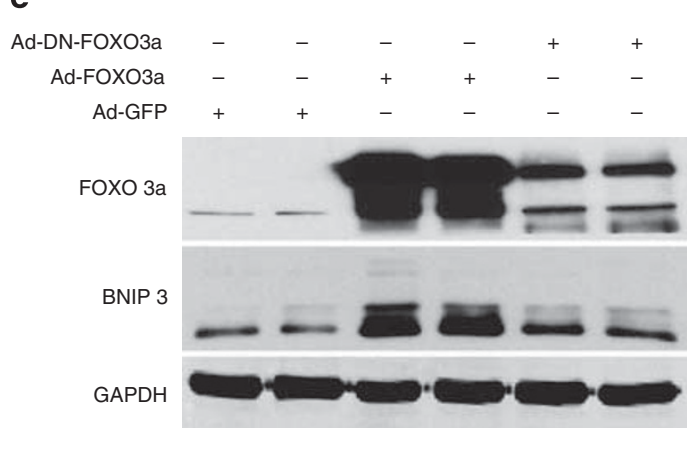

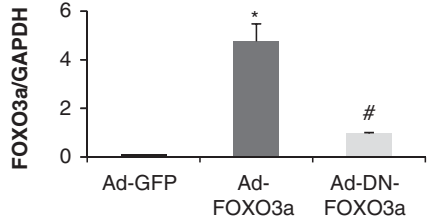

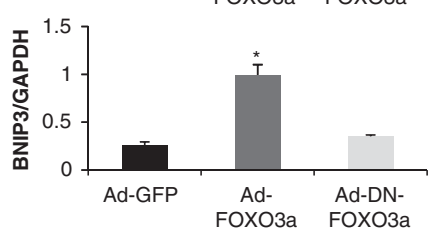

$\mathbf{f}$
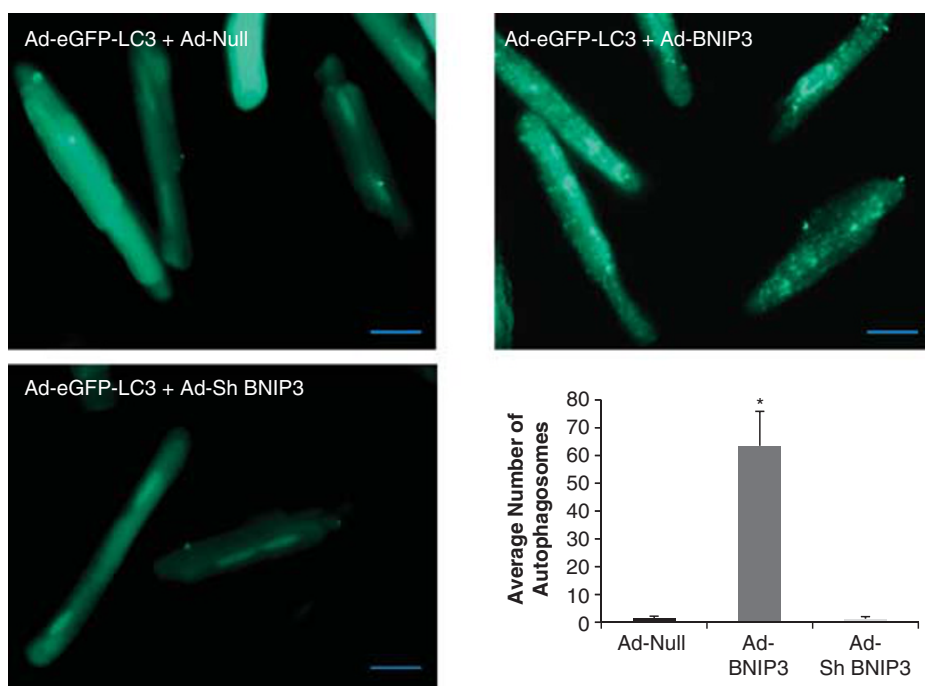

g
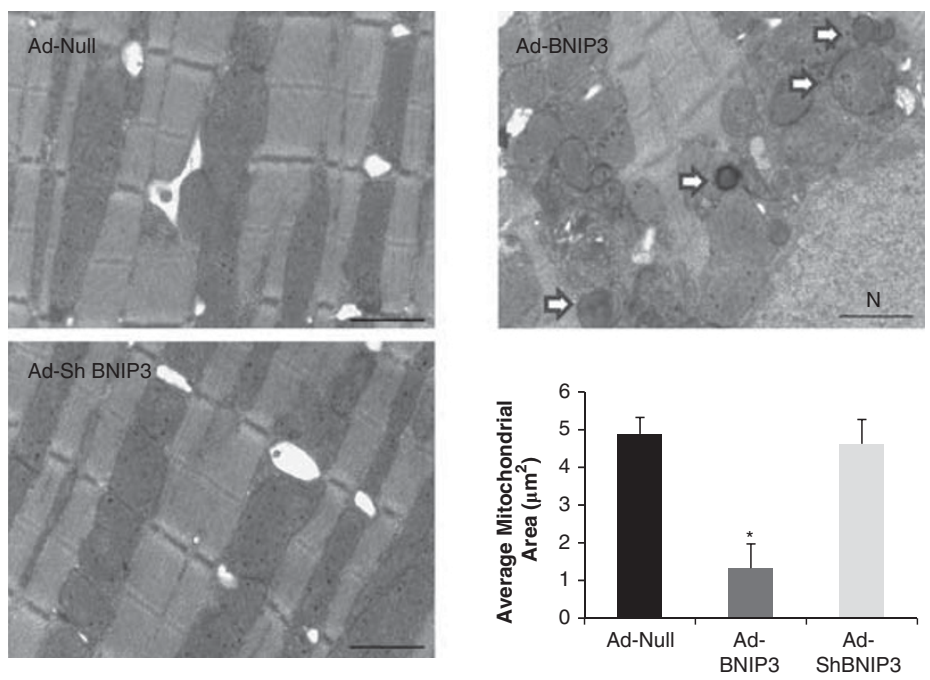

Figure 1 BNIP3 is upregulated $2 \mathrm{~h}$ after cardiomyocyte stress with PE or calcium and is inhibited by $3 \mathrm{MA}$. (a) Western blotting analysis of protein lysates from adult cardiomyocytes (ACM) stressed with PE or Calcium. BNIP3 expression was significantly upregulated $2 \mathrm{~h}$ after ACM stress with PE or calcium and with chloroquine (Chl) treatment, ${ }^{*} P<0.05$ versus $C T L$ and 3 MA-treated samples, ${ }^{\#} P<0.05$ versus $C T L+C h l$. (b) BNIP3 mRNA significantly increased with $P E$ treatment, ${ }^{*} P<0.05$ versus CTL. 3 MA inhibited the increase in BNIP3 mRNA in PE-stressed cardiomyocytes, ${ }^{\sharp} P<0.05$ versus PE. (c) Ultrastructurally, autophagolysosomes (white arrows) were observed in the Chl, $\mathrm{PE}$ and the $\mathrm{PE}+$ chl-treated cardiomyocytes. $3 \mathrm{MA}$ treatment inhibited the formation of autophagolysosomes in PE-stressed cardiomyocytes, images $\times 12000$ magnified, scale bar $1 \mu \mathrm{m}$. (d) $\mathrm{Chl}$ increased the number of autophagosomes in normal cradiomyocytes, ${ }^{*} P<0.05$ versus $\mathrm{CTL}$ and $3 \mathrm{MA}$. There was also increase in the number of autophagosomes in PEstressed cardiomyocytes for $2 \mathrm{~h}$ with the highest number of autophagosomes observed in the PE $+\mathrm{Chl}$ stressed cardiomyocytes, ${ }^{\#} P<0.05$ versus CTL and PE $+3 \mathrm{MA}$, ${ }^{\&} P<0.05$ versus all other groups. Scale bar $100 \mu \mathrm{m}$. (e) The overexpression of constitutive active FOXO3a (Ad-FOXO3a) increased BNIP3 expression in ACM in vitro * $P<0.05$ versus Ad-GFP and Ad-DN-FOXO3a. (f) BNIP3 overexpression robustly increased the number of autophagososmes, ${ }^{\star} P<0.05$ versus Ad-Null and Ad-Sh BNIP3, Scale bar $50 \mu \mathrm{m}$. (g) Ultrastructurally, BNIP3 overexpression robustly decreased the average mitochondrial area, $P<0.05$ versus Ad-Null and Ad-Sh BNIP3. Arrows showing autophagosomes. Images $\times 12000$ magnified, scale bar $1 \mu \mathrm{m}$. All experiments were preformed three times with comparable results. N, nucleus 
been shown that JNK potentiates AKT activity via its phosphorylation at Thr450 and increases the interaction of AKT with its upstream effectors, PI3K1 and PDK. ${ }^{20}$ Phosphorylated JNK and AKT are significantly increased in heart failure compared with age-matched control. However, and despite the increases in AKT activity and the phosphorylation of FOXO3a at Ser253, there were significant increases in FOXO3a effectors, BNIP3 and MurF-1 in the HF Placebo a
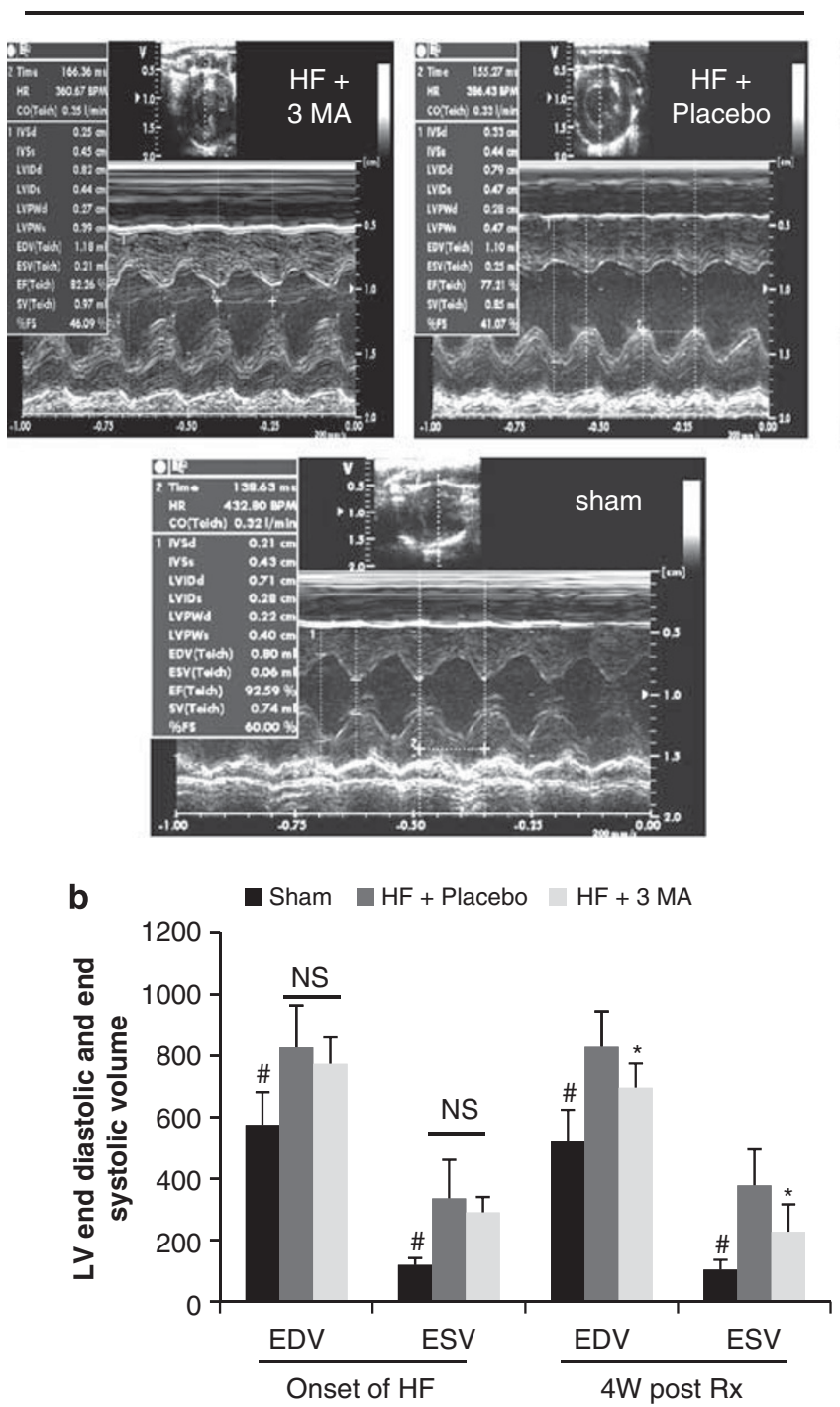

d
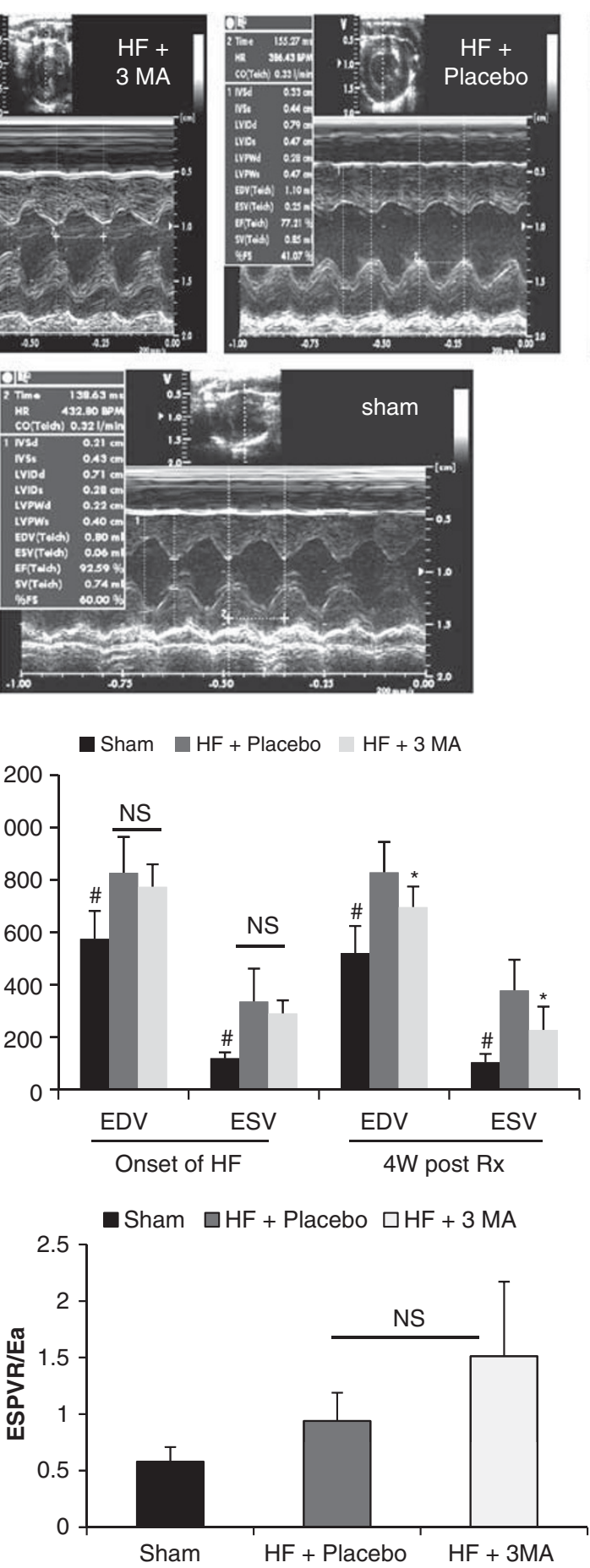
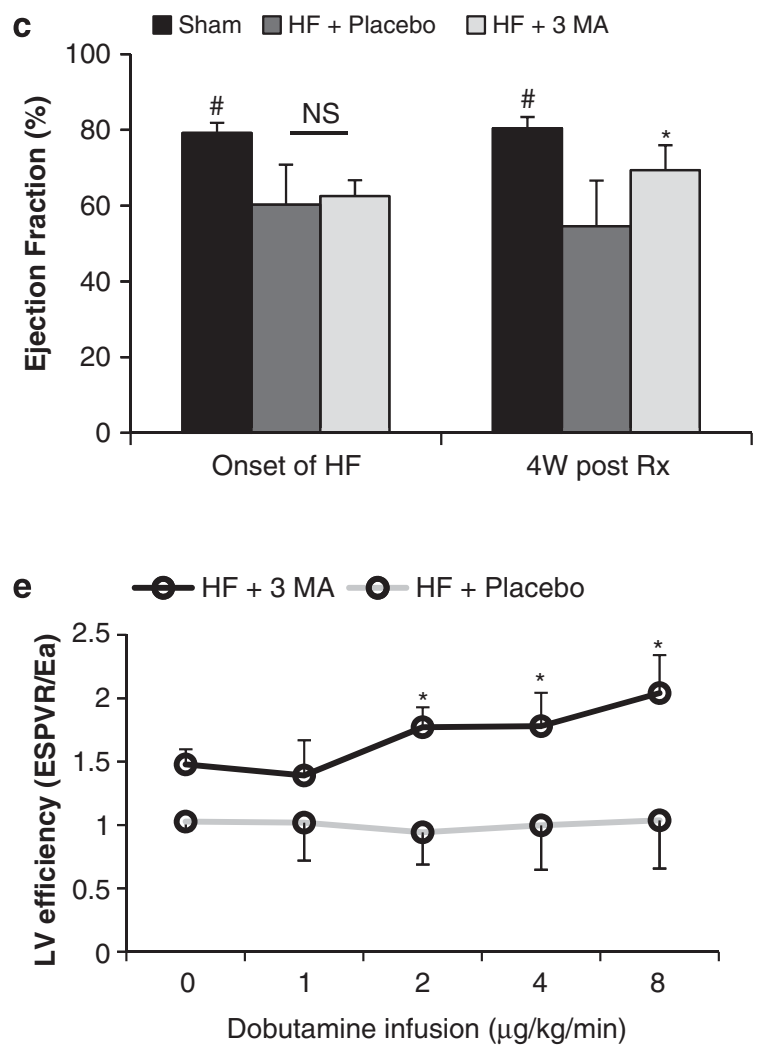

Figure 2 (Continued) 


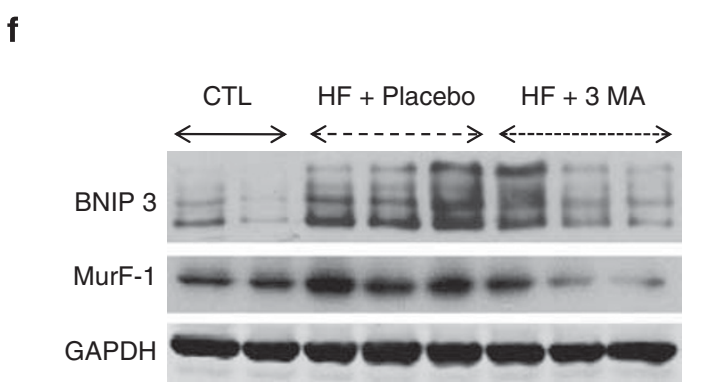

g

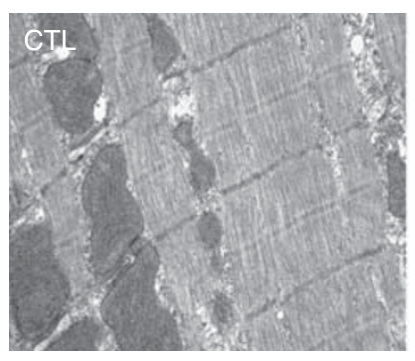

h
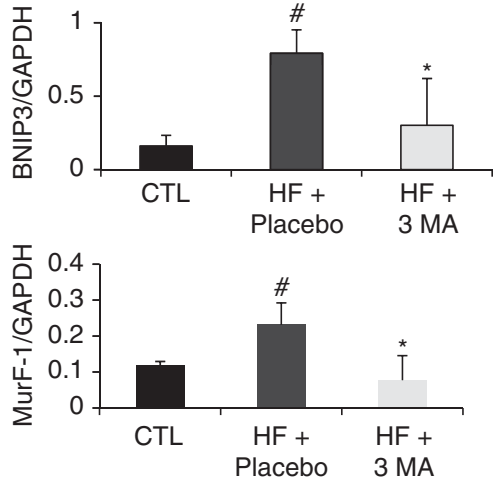

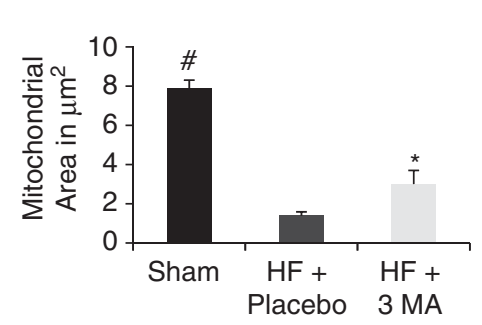

i $\triangle \longrightarrow$ Mito Area in $\mu \mathrm{m}^{2} \longrightarrow$ BNIP3

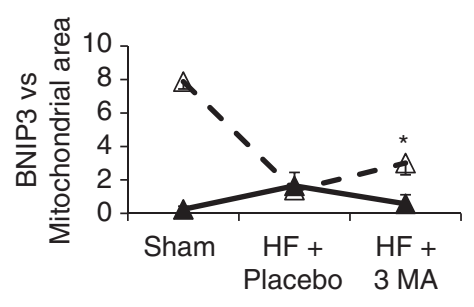

Figure 23 MA reversed cardiac remodeling and attenuated BNIP3 expression in heart failure. (a) M-mode images of the sham placebo and the 3 MA-treated groups at the onset of heart failure and 1 month after treatment. ( $\mathbf{b}$ and $\mathbf{c}$ ) There was a significant decrease in left ventricular end diastolic and end systolic volume, as well as a significant increase in ejection fraction 1 month after $3 \mathrm{MA}$ treatment compared with placebo, ${ }^{\sharp} P<0.05$ versus $\mathrm{HF}+3 \mathrm{MA}$ and $\mathrm{HF}+\mathrm{Placebo},{ }^{*} P<0.05$ versus $\mathrm{HF}+\mathrm{Placebo}$. (d and $\left.\mathbf{e}\right)$ Hemodynamically, there was a trend in improved left ventricular efficiency in the $3 \mathrm{MA}$-treated group; however, there was a significant increase in left ventricular contractility and efficiency in response to $\beta$-adrenergic stimulation with dobutamine, ${ }^{*} P<0.05$ versus HF + placebo. (f) Western blot analysis of LV tissue lysate from control, $\mathrm{HF}+$ Placebo and HF $+3 \mathrm{MA}$ groups. BNIP3 and MurF-1 expression were significantly increased in $\mathrm{HF},{ }^{\#} P<0.05$ versus CTL. 3 MA treatment significantly decreased the expression of FOXO3a effectors BNIP3 and MurF-1, ${ }^{*} P<0.05$ versus HF + Placebo. (g) Ultrustructurally, 3MA attenuated the presence of autophagolysosomes and restored mitochondrial cristae; whereas, the placebo group had severely defragmented mitochondria with the presence of autophagolysosomes (white arrows), images $\times 12000$ magnified. (h) 3 MA significantly improved mitochondrial area, ${ }^{*} P<0.05$ versus $\mathrm{HF}+$ Placebo, ${ }^{\#} P<0.05$ versus $\mathrm{HF}+$ Placebo and $\mathrm{HF}+3 \mathrm{MA}$. (i) showing the correlation between mitochondrial area and BNIP3 expression. 3 MA decreased BNIP3 expression in heart failure and significantly improved the area per unit mitochondrion, ${ }^{\star} P<0.05$ versus $\mathrm{HF}+$ Placebo

group. The long-term administration of $3 \mathrm{MA}$ in vivo attenuated JNK activity and significantly decreased the expression of $\mathrm{FOXO} 3$ a effectors despite the decreases in AKT activity and the decrease in FOXO3a phosphorylation by AKT Figure 3a. From the above, we hypothesized that the long-term activation of JNK opposes the inhibitory effect of AKT on FOXO3a, via its phosphorylation at sites that are yet to be identified, and thus override the inhibitory effect of AKT on FOXO3a for the activation of the latter, promoting the transcription of FOXO3a effectors, BNIP3 and MurF-1, respectively. In other words, the phosphorylation of FOXO3a by AKT is dependent on JNK activity and that prolonged JNK signaling dominate over AKT signaling and promotes or activates $\mathrm{FOXO} 3 \mathrm{a}$, despite its phosphorylation by AKT, for the transcription and the expression of its effectors BNIP3 and MurF-1, respectively. Moreover, prolonged JNK activity phosphorylates $\mathrm{Bcl}-2$ and leads to the increase in Bax to Bcl-2 ratio Figure 3b. 3 MA did not affect ERK or P38 activity in vivo Figure 3b. In order to prove the above hypothesis, we used an Ad-DN-JNK that was delivered via a cross-clamp technique followed by ascending aortic banding (AAB). Saline and an Ad-GFP were used as controls. Transthoracic echocardiography data and M-mode images are shown in the Supplementary Table 3 and Figure $4 a$, respectively. There were significant decreases in the heart weight to body weight ratio as well as in the septal and posterior wall thickness and the LV end systolic diameter and volume of the Ad-DN-JNK group compared with the 
a
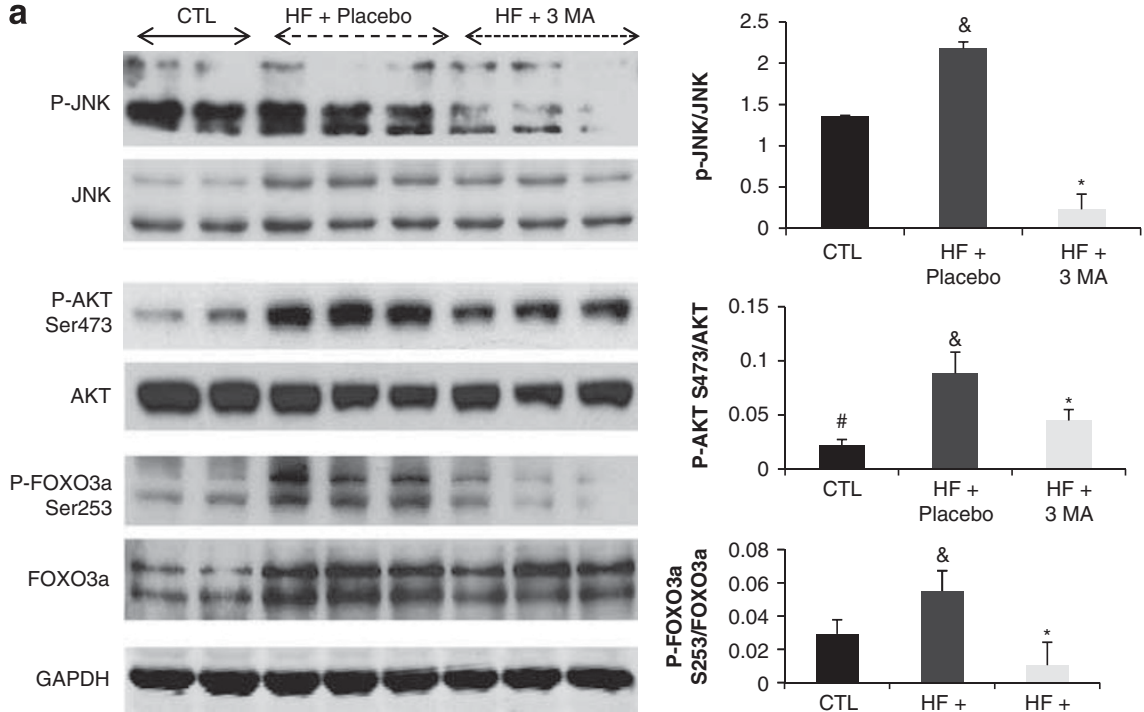

b
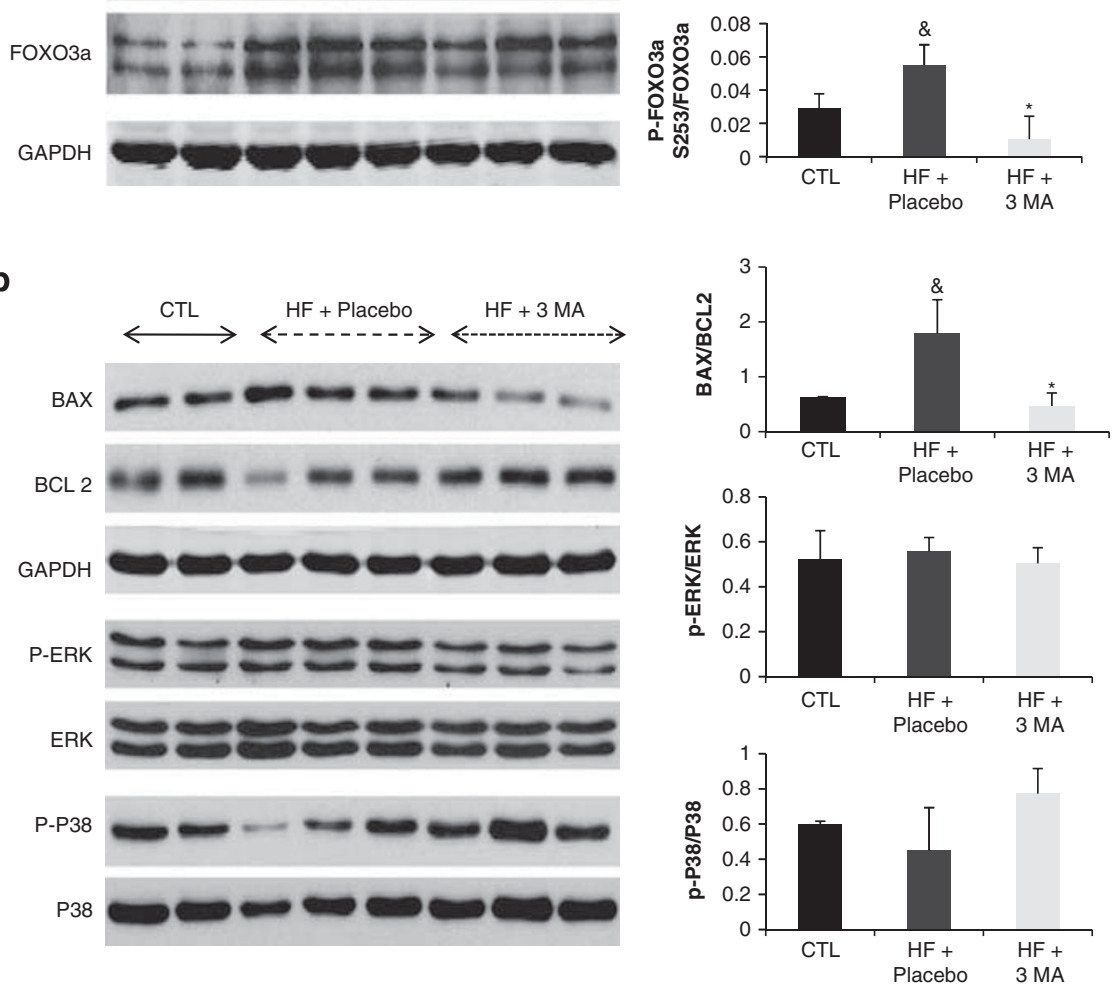

Figure 33 MA significantly attenuated JNK and AKT activities and decreased Bax to Bcl-2 ratio in HF. (a) Western blot analysis of LV tissue lysate from control, $\mathrm{HF}+$ Placebo and HF + 3 MA groups. JNK activity, AKT activity and phospho-FOXO3a at Ser253 were significantly increased in $\mathrm{HF},{ }^{\&} P<0.05$ versus CTL. $3 \mathrm{MA}$ significantly decreased JNK and AKT activities and thus decreased the phosphorylation of FOXO3a at ser $253,{ }^{*} P<0.05$ versus $\mathrm{CTL}$ and $\mathrm{HF}+\mathrm{Placebo}$, ${ }^{\#} P<0.05$ versus $\mathrm{HF}+\mathrm{Placebo}$ and $\mathrm{HF}+3 \mathrm{MA}$. (b) The ratio of $\mathrm{Bax}$ to $\mathrm{Bcl}-2$ is increased in $\mathrm{HF},{ }^{\&} P<0.05$ versus $\mathrm{CTL}$. $3 \mathrm{MA}$ significantly decreased $\mathrm{Bax}$ to $\mathrm{Bcl}-2$ ratio, ${ }^{*} P<0.05$ versus $\mathrm{HF}+\mathrm{Placebo}$. There was no difference in ERK and P38 phosphorylation between the three groups

Saline and the Ad-GFP groups, Figures 4b-d. This reflected significant increases in LV fractional shortening and ejection fraction, Figure $4 \mathrm{e}$. On the molecular level, the decrease in JNK activity using Ad-DN-JNK, prevented the significant increase in AKT activity and the increase in FOXO3a phosphorylation by AKT in response to pressure overload as shown in the Saline and the Ad-GFP groups, Figure $4 \mathrm{f}$. Moreover, the continuous decrease in JNK activity, for 2 weeks, inhibited the increase in BNIP 3 expression, despite the decrease in AKT activity, and inhibited the increases in LC3-2 to LC3-1 ratio and Bax to Bcl-2 ratio in response to pressure overload as shown in the Saline and Ad-GFP groups, respectively Figure $4 \mathrm{f}$.
FOXO3a effectors, BNIP3 and MurF-1, are significantly upregulated in $\mathrm{POH}$ and peak in heart failure. In order to understand the timing at which this signaling pathway is altered in response to pressure overload, we conducted a kinetic study and examined the expression of the key components in the pathway at 1 week and 3 weeks of $\mathrm{POH}$ and in heart failure. Echo data are shown in the Supplementary Table 4. Our results suggest that JNK and AKT activities are significantly upregulated 1 week after pressure overload and continue to be activated up to heart failure Figure $5 \mathrm{a}$. There were gradual increases in the expression of FOXO3a effectors, BNIP3 and MurF-1, that became significant 2-3 weeks after pressure overload and peaked in heart failure, despite the increases in 
FOXO3a phosphorylation at Ser253 by AKT Figure 5a. These results further validate that the continuous and prolonged JNK activation in response to pressure overload, dominates over AKT signaling and overrides the inhibitory effect of AKT on FOXO3a for the gradual increases in the expression of FOXO3a effectors, BNIP3 and MurF-1, which peak in heart failure. Moreover, the increases in JNK activity significantly increased $\mathrm{Bax}$ to $\mathrm{Bcl}-2$ ratio the first week after pressure overload and continued to be elevated up to heart failure Figure 5a. This signaling pathway has been validated in human samples of heart failure. There are robust increases in JNK and AKT activities as well as significant increase in BNIP3 expression, despite the increase in AKT activity, in human samples of HF Figure 5b.

a
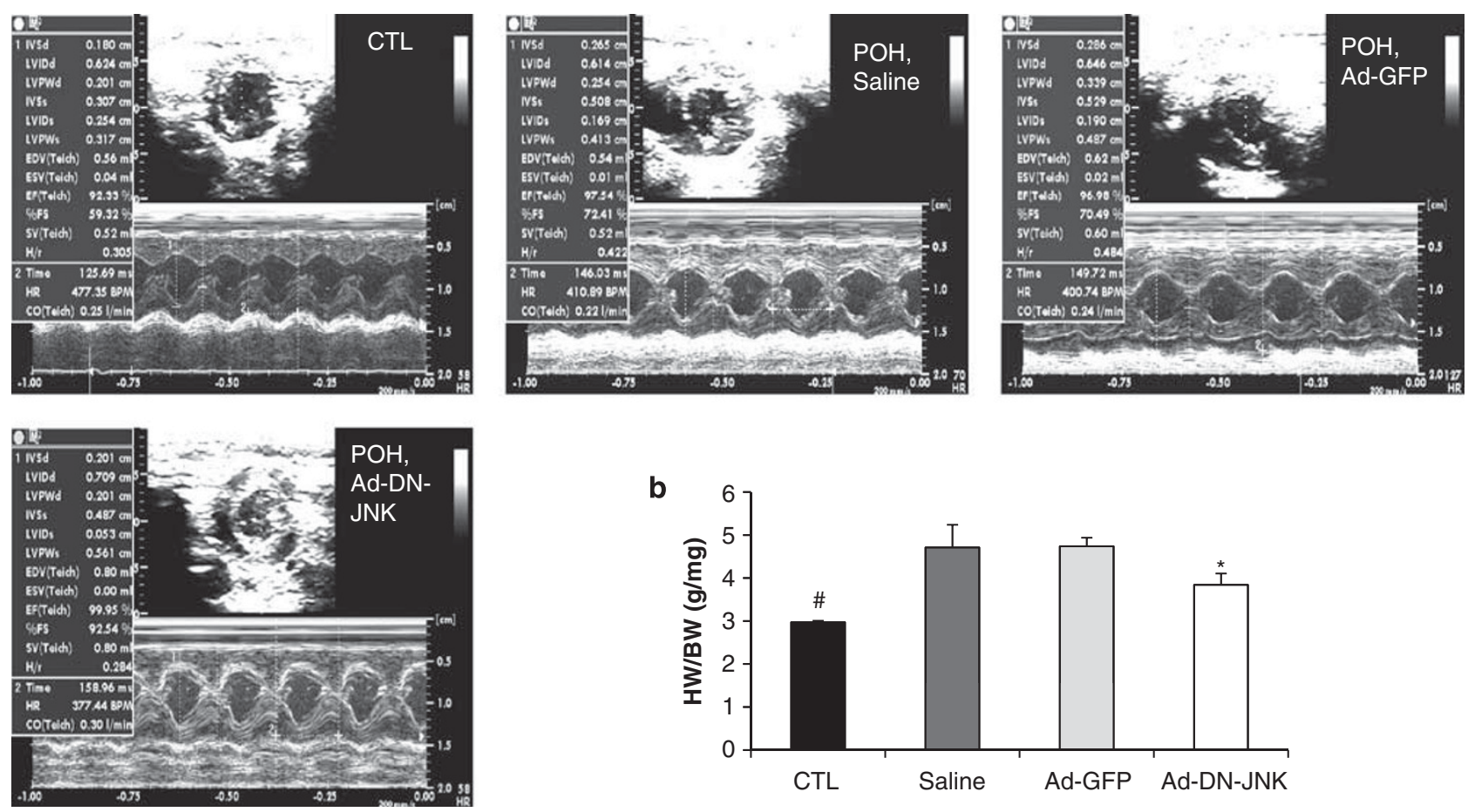

b

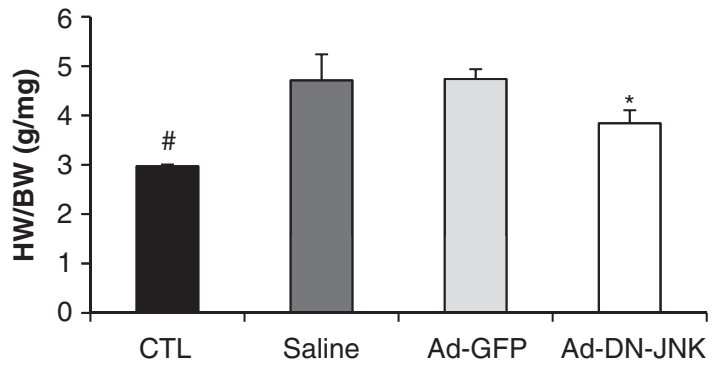

C

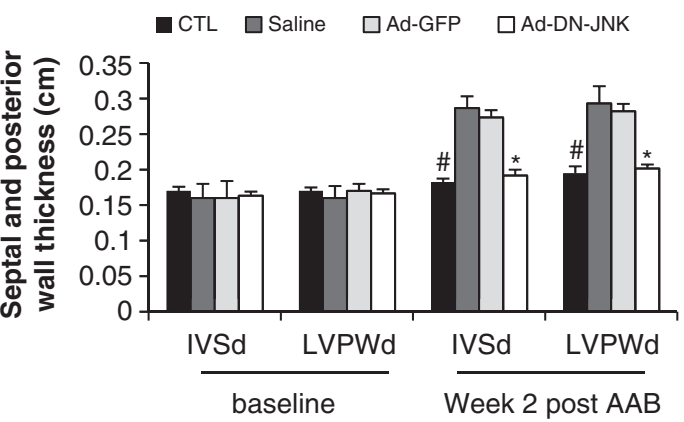

d

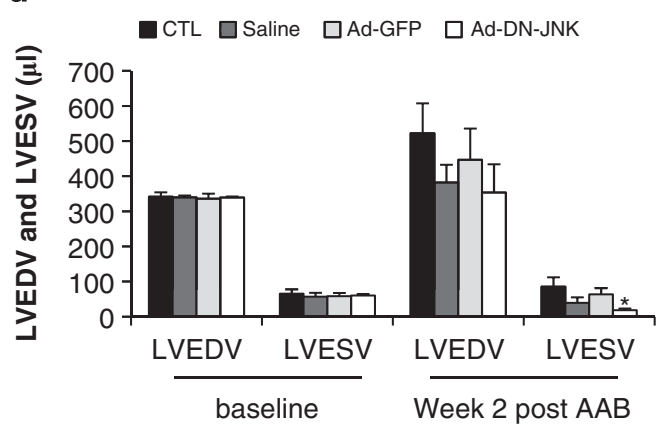

e

$\square$ CTL $\square$ Saline $\square$ Ad-GFP $\square$ Ad-DN-JNK

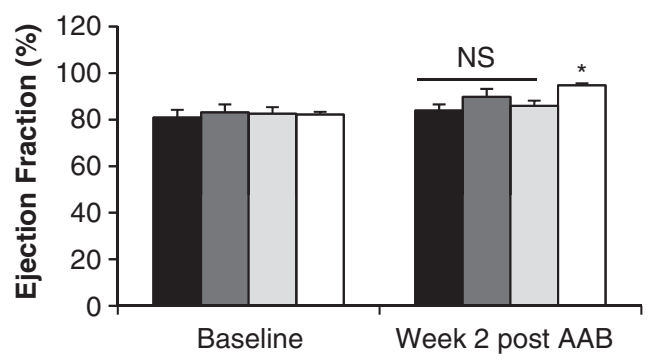

Figure 4 (Continued) 

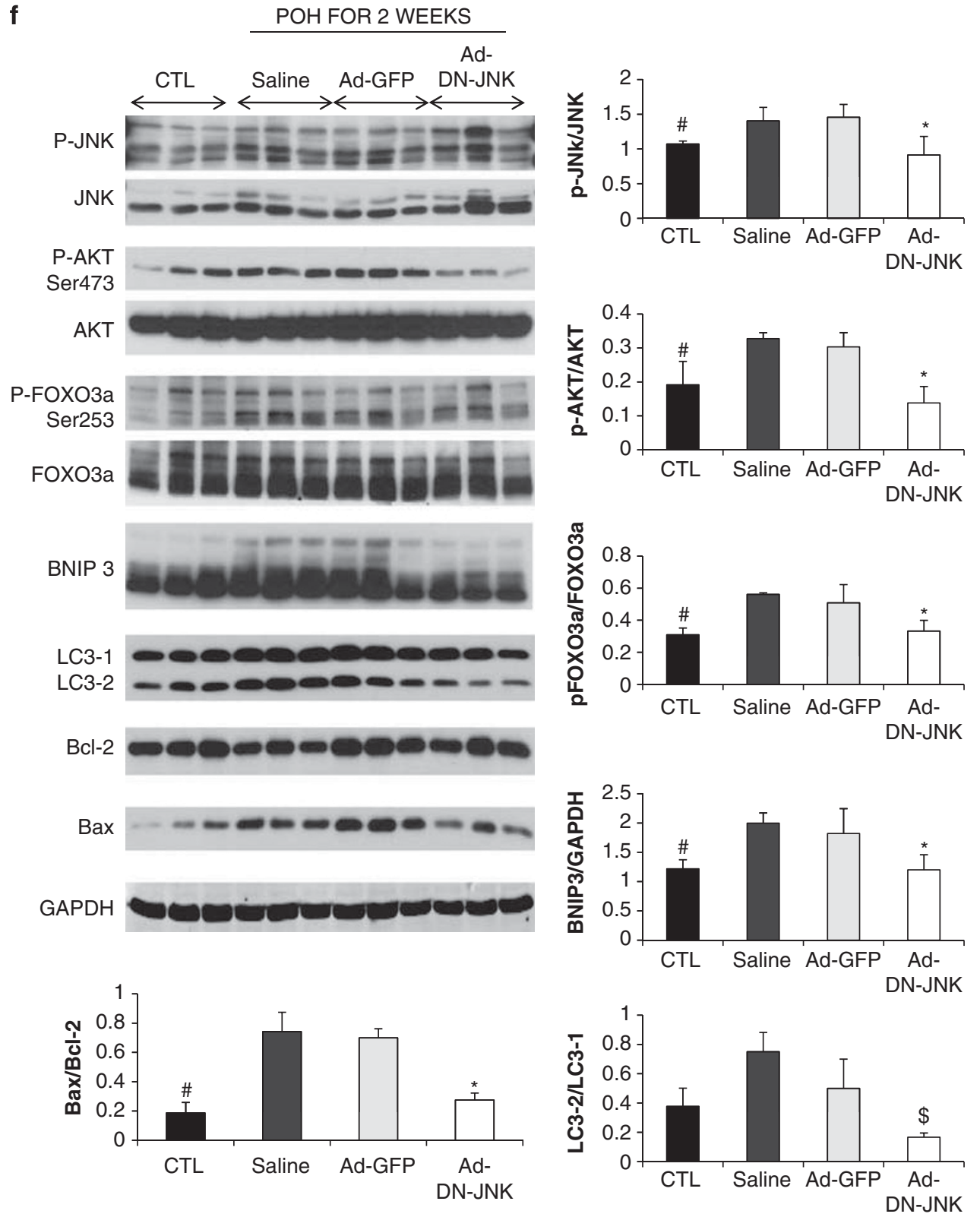

Figure 4 Gene delivery of Ad-DN-JNK inhibited cardiac hypertrophy and enhanced LV contractility and attenuated JNK and AKT activities with decrease in BNIP3 expression and Bax to Bcl-2 ratio in a rat model of POH. (a) Representative M-mode images of CTL, POH + Saline, POH + Ad-GFP and POH + Ad-DN-JNK groups. (b) There was significant decrease in the HW/BW of the Ad-DN-JNK group, ${ }^{*} P<0.05$ versus Saline and Ad-GFP, ${ }^{\#} P<0.05$ versus Saline, Ad-GFP and Ad-DN-JNK. (c and d) There were decreases in the septal and posterior wall thickness and the LVESV in the Ad-DN-JNK group, ${ }^{*} P<0.05$ versus Saline and Ad-GFP, \#P<0.05 versus Saline and Ad-GFP. (e) The EF by echocardiography was significantly higher in the Ad-DN-JNK group, ${ }^{\star} P<0.05$ versus Saline and Ad-GFP. (f) Western blot analysis of $\mathrm{LV}$ tissue lysates from CTL, POH + saline, POH + Ad-GFP and POH + Ad-DN-JNK groups. Ad-DN-JNK significantly attenuated JNK and AKT activities, as well as the phosphorylation of FOXO3a by AKT at Ser253 and prevented the increase in BNIP3 expression and the increase in Bax to Bcl-2 ratio in response to pressure overload, ${ }^{*}$ and ${ }^{\sharp} P<0.05$ versus saline and Ad-GFP. Ad-DN-JNK decreased the conversion of LC3-1 to LC3-2 in response to pressure overload, ${ }^{\$} P<0.05$ versus CTL, saline and Ad-GFP

Ultrastructurally, there was an increase in autophagosomes the first week after $\mathrm{POH}$, which continued to be present up to heart failure Figure $6 \mathrm{a}$. There was a significant decrease in the average area per mitochondrion the first week after $\mathrm{POH}$. The mitochondrial area declined further 3 weeks after pressure overload and was the lowest in heart failure Figures $6 a$ and $b$. The gradual and significant increase in BNIP3 expression in $\mathrm{POH}$ up to heart failure reflected a gradual and significant decrease in the average area per mitochondrion, which was the lowest in heart failure where BNIP3 expression was the highest Figure 6c.

\section{Discussion}

$\mathrm{BNIP} 3$ is a $\mathrm{BH} 3$ protein that promotes mitochondrial destruction and signals mitochondrial death via the opening of 
a
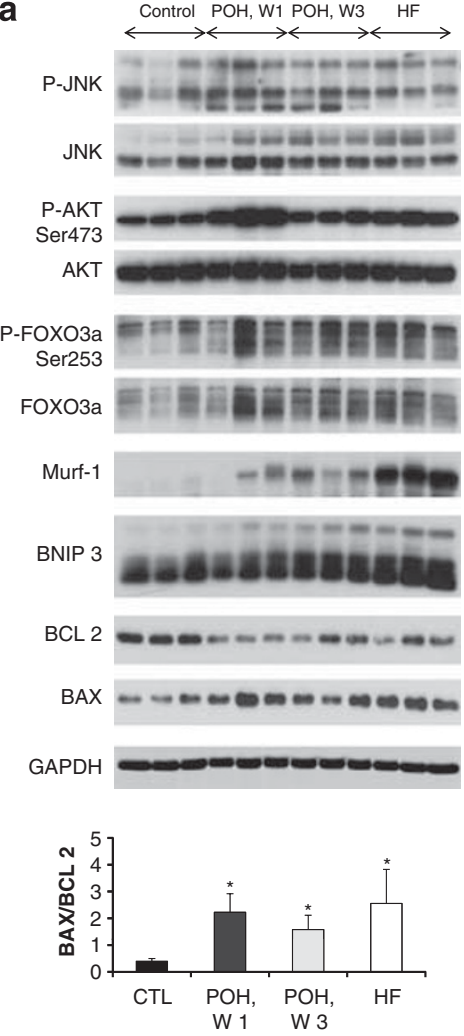
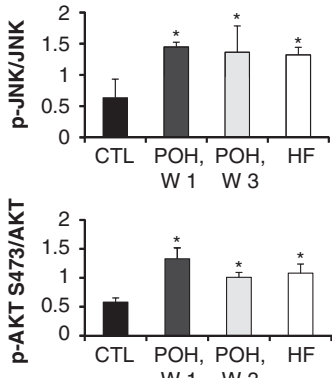

W 1 W 3
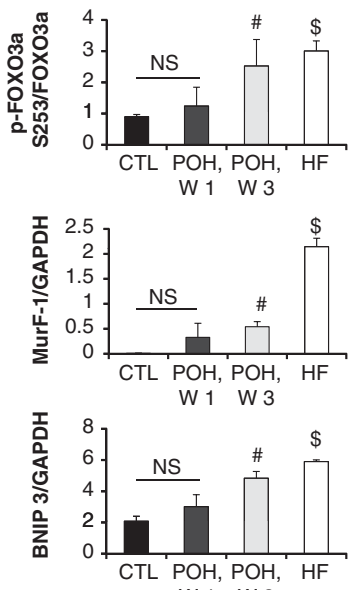

b

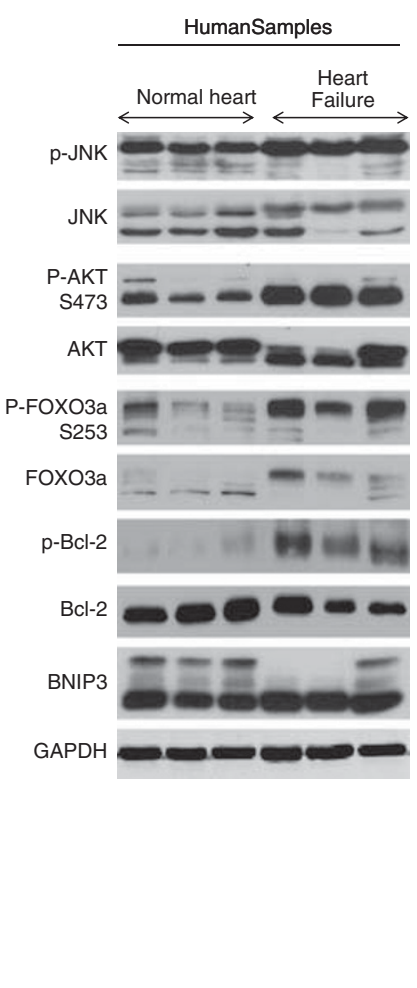

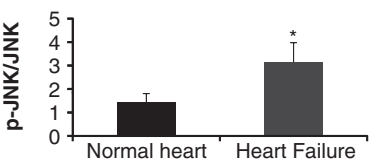
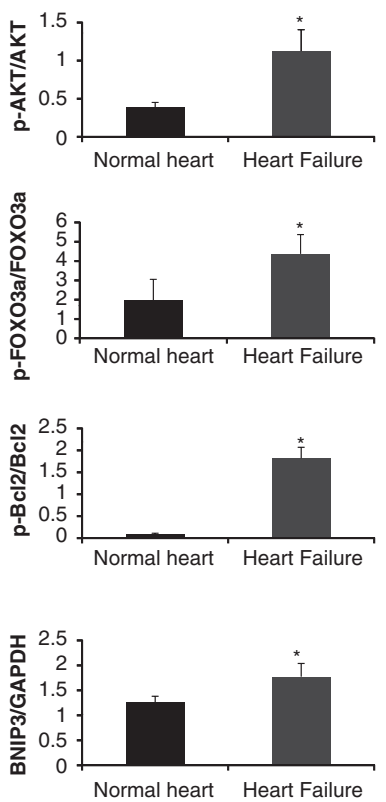

Figure 5 The FOXO3a effectors, BNIP3 and MurF-1, are significantly upregulated in $\mathrm{POH}$ and peaked in heart failure. (a) Western blot analysis of LV tissue lysates from CTL, Week $1 \mathrm{POH}(\mathrm{POH}, \mathrm{W} 1)$, Week $3 \mathrm{POH}(\mathrm{POH}, \mathrm{W} 3)$ and HF samples. JNK and AKT activities as well as Bax to Bcl-2 ratio are significantly increased 1 week after POH and remained to be elevated up to heart failure, ${ }^{*} P<0.05$ versus CTL. The phospho-FOXO3a at Ser253 and the expression of BNIP3 and MurF-1 were significantly increased 3 weeks after pressure overload and peaked in heart failure, ${ }^{\#} P<0.05$ versus $C T L$ and $P O H, W 1,{ }^{\$} P<0.05$ versus all the three groups. (b) Western blot analysis of $L V$ tissue lysates from normal and failing human hearts. JNK and AKT activities as well as phospho-FOXO3a Ser253 and phospho-Bcl-2 and BNIP3 expression are robustly increased in heart failure, ${ }^{*} P<0.05$ versus $\mathrm{CTL}$

mitochochondrial permeability transition pore and the subsequent release of cytochrome $\mathrm{C}^{11}$ Moreover, it is an autophagy receptor that activates mitophagy in a noncanonical order via the binding and the lipidation of LC3. ${ }^{12}$ Our data suggest that BNIP3 expression is significantly upregulated $2 \mathrm{~h}$ after cardiomyocyte stress in vitro with $\mathrm{PE}$ or calcium. Also, the addition of chloroquine to the cultured medium increased BNIP3 expression, whereas 3 MA attenuated its expression in stressed cardiomyocytes. The overexpression of BNIP3 in cardiac myocytes, using Ad-BNIP3, robustly increased autophagosomes formation and significantly decreased mitochondrial area. In cardiac myocytes and in skeletal muscles, BNIP3 is the effector of the transcription factor FOXO3a. ${ }^{14,21}$ The activation of FOXO3a or its inhibition is dependent on its post-translational modification status. ${ }^{22}$ AKT phosphorylates FOXO3a at Thr32, Ser253 and Ser315 leading to its inhibition, whereas JNK interacts and phosphorylates FOXO3a, at sites that are still unknown, leading to its activation. ${ }^{23}$

In heart failure, FOXO3a effectors, BNIP3 and MurF-1 are highly expressed compared with age-matched controls. The increase in BNIP3 expression leads to increase in mitochondrial destruction, mitochondrial apoptosis and mitophagy; whereas, the increase in MurF-1 and atrogin-1 promote cardiac atrophy. ${ }^{24-26} 3 \mathrm{MA}$ treatment robustly decreased the expression of FOXO3a effectors, BNIP3 and MurF-1, respectively, and hence attenuated mitochondrial damage and cardiac atrophy by interfering with JNK signaling. In our model of heart failure, both JNK and AKT were activated; however, AKT failed to inhibit FOXO3a. On the other hand, 3 MA by attenuating JNK activity reversed this signaling pathway and decreased the expression of FOXO3a effectors and pro-apoptotic marker Bax and, hence, decreased Bax to $\mathrm{Bcl}-2$ ratio. These results led us to hypothesize that prolonged JNK signaling dominates over AKT and overrides the inhibitory effect of AKT on FOXO3a and forces the latter to re-translocate into the nucleus for the transcription of its effectors. Moreover, prolonged JNK signaling phosphorylates $\mathrm{Bcl}-2$ and increases the expression of the pro-apoptotic marker Bax, thus increasing Bax over Bcl-2 ratio and cardiomyocyte loss through apoptosis. This hypothesis was further validated using an adenovirus expressing dominant negative JNK that was delivered in vivo using a crossclamp technique followed by aortic constriction for the creation of pressure overload. The continuous inhibition of JNK activation for 2 weeks prevented cardiac hypertrophy and prevented the decline in cardiac function in response to pressure overload. These results are consistent with those of 
a
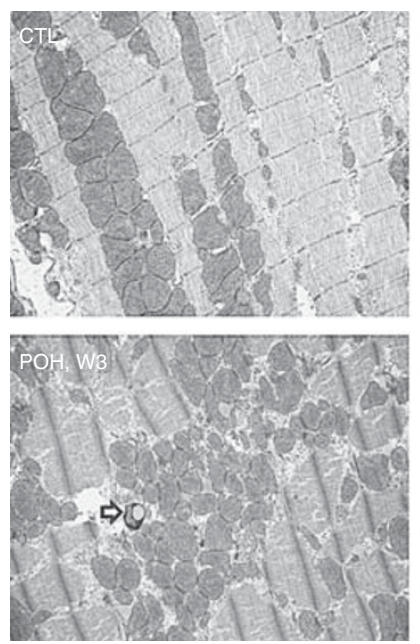

b

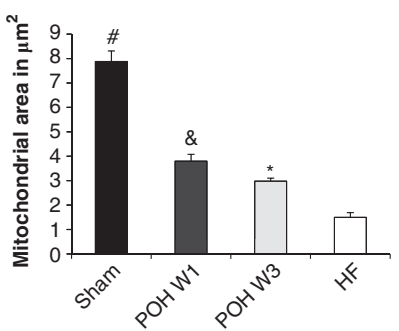

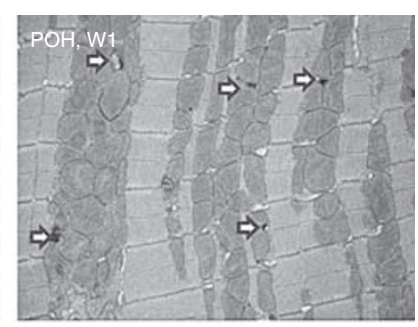

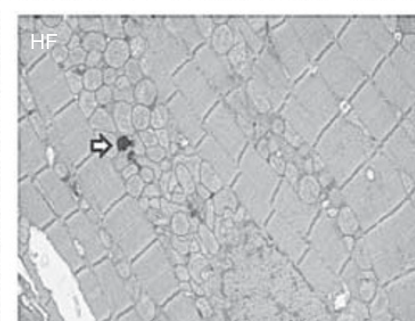

C

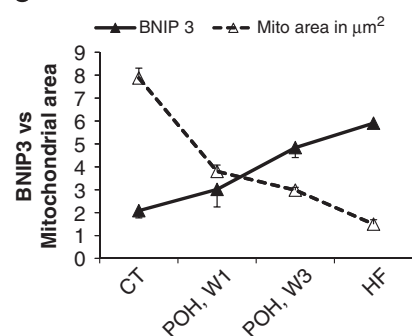

Figure 6 The Mitochondrial area declines in $\mathrm{POH}$ and is the lowest in heart failure. (a) TEM images of CTL, 1-3 weeks after $\mathrm{POH}$ and HF. The mitochondrial area gradually declines in $\mathrm{POH}$ and is the lowest in HF. White arrows showing the presence of autophagolysosomes, images $5000 \times$ magnified. (b) mitochondrial area in CTL, POH W1, POH W3 and in HF, ${ }^{\#} P<0.05$ versus CTL, POH W1, POH W3 and HF, ${ }^{\&} P<0.05$ versus $\mathrm{POH}$ W3 and $\mathrm{HF},{ }^{*} P<0.05$ versus HF. (c) Showing the correlation between BNIP3 expression and mitochondrial area. The mitochondrial area is inversely proportional to the increase in BNIP3 expression

Choukroun et al. ${ }^{27,28}$ who have shown that the inhibition of JNK suppresses cardiac hypertrophy in vitro and in vivo in response to endothelin and pressure overload, respectively. On the molecular level, continuous JNK inhibition for 2 weeks by Ad-DN-JNK prevented the increase in AKT activity, BNIP3 expression and the increase in $\mathrm{Bax}$ to $\mathrm{Bcl}-2$ ratio in response to pressure overload Figure 7.

Our kinetic study shows that JNK and AKT signaling pathways are activated early in the first week of $\mathrm{POH}$ and remains active throughout compensated hypertrophy up to heart failure. However, what is more interesting is that the prolonged and persistent JNK signaling leads to the gradual but significant rise in FOXO3a transcription factors, which peak in heart failure despite the increase in AKT activity and the increase in FOXO3a phosphorylation at Ser253 by AKT. This signaling pathway was further validated in human samples of heart failure. The steady and progressive increase in BNIP3 expression leads to progressive and steady increase in mitochondrial fragmentation and to the gradual decrease in the average area per unit mitochondrion, which is the lowest in heart failure. On the other hand, the attenuation of BNIP3 expression by 3 MA decreased mitochondrial fragmentation and increased the average area per mitochondrion.

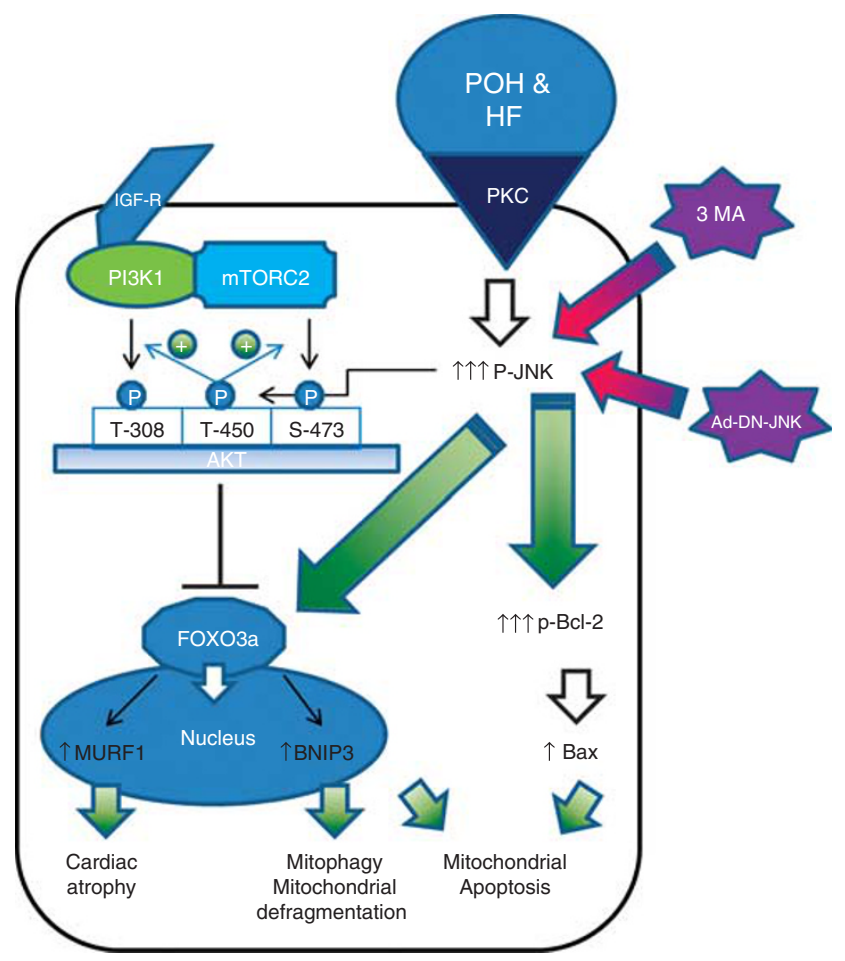

Figure 7 Chronic JNK signaling dominates over AKT signaling and overrides the inhibitory effect of AKT on FOXO3a for the transcription of its effectors, BNIP3 and MurF-1 in POH and in heart failure. JNK and AKT signaling are activated in response to pressure overload. However, despite the increase in AKT activity, BNIP3 expression increased significantly in $\mathrm{POH}$ and peaked in HF. On the other hand, the decrease in JNK activity by Ad-DN-JNK and 3 MA in vivo decreased AKT activity and decreased the expression of FOXO3a effectors, BNIP3 and MurF-1. In other words, the expression of FOXO3a effectors, BNIP3 and MurF-1, are directly related to JNK activity and that the chronic activation of JNK dominates over AKT signaling for the activation of $\mathrm{FOXO} 3 \mathrm{a}$ and for the transcription of its effectors, BNIP3 and MurF-1. Moreover, the increase in JNK activity phosphorylates Bcl-2 and increases $\mathrm{Bax}$ to $\mathrm{Bcl}-2$ ratio, further promoting mitochondria-induced apoptosis. Green arrows: promote signaling pathway, Red arrows: inhibit signaling pathway

\section{Conclusion}

BNIP3 induces mitochondrial autophagy and apoptosis early on in $\mathrm{POH}$ and is further increased in heart failure. JNK is a critical regulator of BNIP3 expression in vivo. Prolonged JNK signaling dominates and overrides AKT signaling and activates $\mathrm{FOXO} 3 \mathrm{a}$ contributing to the gradual increase in BNIP3 expression in compensated hypertrophy, which peaks in heart failure. The gradual increase in BNIP3 expression causes destruction of the mitochondria and promotes mitochondrial-induced apoptosis and mitophagy leading to the gradual decline in cardiac function and LV remodeling. $3 \mathrm{MA}$ modulates the expression of BNIP3, in vivo, via the interference with JNK signaling and reverses cardiac remodeling in heart failure.

\section{Materials and Methods}

Isolation and culture of adult rat cardiomyocytes and in vitro experiments. Adult rat ventricular cardiomyocytes were isolated from male Sprague-Dawley rats weighing $(250-350 \mathrm{~g})$ as previously described. ${ }^{29,30}$ Cells were stressed for $2 \mathrm{~h}$ with $10 \mu \mathrm{M}$ of PE or with the addition of $2 \mathrm{mM}$ of calcium to the culture medium. Chloroquine was added at a concentration of $0.5 \mu \mathrm{M}$ for 
autophagosome-lysosome fusion inhibition, whereas autophagy was inhibited by the addition of $2.5 \mathrm{mM}$ of $3 \mathrm{MA}$ to the cultured medium. All reagants were purchased from (Sigma Chemicals, St. Louis, MO, USA). After $2 \mathrm{~h}$, the cells were lysed using RIPA buffer as mentioned in the western blotting section $(n=3$ for each experiment in vitro). For electron microscopy, cells were fixed with $3 \%$ glutarldehyde for $24 \mathrm{~h}$ at $4{ }^{\circ} \mathrm{C}$. The next day, the cells were detached using a scraper and were sent for electron microscopy for processing as discussed below in the electron microscopy section. For the Autophagic flux experiments, cells were infected with adenovirus eGFP-LC3 and images were taken using fluorescent microscope, Olympus $1 \times 71$.

Western blotting. $30 \mathrm{ug}$ of proteins were loaded and electrophoresed using SDS-PAGE gels then transferred to a PVDF membrane. The membrane was blocked for $1 \mathrm{~h}$ using blocking solution then was incubated with the primary $A b$ overnight at $4{ }^{\circ} \mathrm{C}$. The following antibodies were used: GAPDH (Sigma, 1: 10000 dilution), LC 3 , BNIP3, JNK, AKT, p-AKT Ser473, FOXO3a, FOXO3a Ser253, Bax, Bcl-2, ERK, p-ERK, P38 and p-P38 (Cell Signaling, Danvers, MA, USA, 1: 1000 dilution), p-JNK (Promega, Madison, WI, USA, 1: 2000 dilution), MurF-1 (BDM Biosciences, Franklin Lakes, NJ, USA, 1: 1000 dilution) and BNIP3 human-specific (Abcam, Cambridge, MA, USA, 1: 1000 dilution). The second day, after three washing steps with TBS$0.05 \%$ Tween-20, the blot was incubated with secondary horseradish peroxidaseconjugated antibody $(1: 10000$ dilution) for $45 \mathrm{~min}$. The blot was washed three times with TBS- $0.05 \%$ Tween-20, then a supersignal west pico chemiluminescent substrate (Thermo Scientific, Barrington, IL, USA) was used for the detection of protein bands using the film method. Bands densities were quantified using photoshop program and were normalized to GAPDH to correct for variations in protein loading.

Quantitative RT-PCR. Total RNA was isolated using RNeasy Protect Mini kit (Qiagen, Hilden, Germany). Reverse transcription was performed using High Capacity cDNA Reverse Transcription Kits (Applied Biosystems, Carlsbad, CA, USA) with random oligo-dT priming as followed by the manufacturer's protocols. PCR was performed using an ABI PRISM Sequence Detector System 7500 (Applied Biosystems) with SYBR Green (BioRad, Hercules, CA, USA) as fluorescent, and ROX (Takara, Otsu, Japan) as a passive reference dye. The PCR primers used were: BNIP3-F, 5'-AGCATGAATCTGGACGAAGC-3' and BNIP3-R 5'-AACATTTTCTGGCCGACTTG-3'; 18S-F, 5' -tgcggaaggatcattaacgga-3' and $18 \mathrm{~S}-\mathrm{R}, 5^{\prime}$-agtaggagaggagcgagcgacc- $3^{\prime}$ was used as an endogeneous-loading control.

Human heart samples. Left ventricular samples were obtained from explanted human hearts obtained at the time of cardiac transplantation. Nonfailing hearts, used as controls, were obtained from donors who died from neurological diseases or motor-vehicle accidents and who had normal cardiac function. The three donors had a median age of 62 . The heart-failure patients had a median age of 60 and their average ejection fraction was $20 \pm 3 \%$.

Electron microscopy. Cells and fractions $\left(1 \mathrm{~mm}^{3}\right)$ from fresh ventricles were pre-fixed in a solution of $3 \%$ glutaraldehyde overnight at $4{ }^{\circ} \mathrm{C}$, post-fixed in $1 \%$ osmium tetroxide $\left(\mathrm{OsO}_{4}\right)$, dehydrated in an ascending series of alcohols, and embedded in epoxy resin. Ultrathin sections were stained with uranylacetate and lead citrate. Samples were viewed under a transmission electron microscope (HITACHI H-7650, Japan). Images were taken at $1 \mathrm{~K}, 5 \mathrm{~K}$ and $12 \mathrm{~K}$ magnification.

Image analysis using Image $\mathrm{J}$. Mitochondrial area analysis was done using Image-J, a public domain Java image-processing program inspired by NIH. Scale setting and calibration were done using the 'Set Scale and Calibration Menu,' and measurement parameters were selected. The mitochondrial area was summed for each image using 12000 magnified images and the average mitochondrial area per image was calculated in $\mu \mathrm{m}^{2}$ (total area/number of mitochondria) after correction by the magnification factor.

Production of recombinant adenoviruses. Recombinant Ad-GFP was prepared as described previously. ${ }^{31}$ Briefly, The Ad-Easy Adenoviral vector system (Stratagene, La Jolla, CA, USA) was used to generate recombinant adenoviruses. Full-length EGFP gene was subcloned into the pShuttle vector (containing the CDNA for enhanced GFP) under the control of CMV promoter. Viral titers were determined by the plaque assay and the absence of replication-competent adenovirus was confirmed by PCR to assess the wild-type E1 region. A dominantnegative JNK adenovirus (Ad-DN-JNK1) was purchased from Seven Hills
Bioreagents (Cincinnati, OH, USA). Ad-DN-FOXO3a and Ad-FOXO3a were purchased from Vector Biolabs (Philadelphia, PA, USA). Adenovirus Null, BNIP3, Sh BNIP3 and EGFP-LC3 were done at Vector Biolabs. A multiplicity of infection of 100 has been used in all infection experiments in vitro $(n=3$ for each experiment in vitro). For the in vivo experiments, the adenovirus was delivered via a cross clamp technique as described below with an infectious dose of 200 pfu/cell.

Experimental model of $A A B$, cross clamp with $A A B$ and study design. All procedures involving the handling of animals were approved by the Animal Care and Use Committee of the Mount Sinai School of Medicine and adhered with the Guide for the Care and Use of laboratory Animals published by the National Institutes of Health. The aortic banding model was used to generate pressure overload-induced hypertrophy and heart failure. Sprague-Dawley rats weighing $180-200 \mathrm{~g}$ underwent $A A B$, as previously described in detail. ${ }^{32}$ For the in vivo Kinetic study, animals were killed 1-3 weeks after $A A B$ and at heart failure development ( $n=4$ at each time point). Before killing, echocardiography was used to assess LV size and function, whereas the clip placement was verified at the time of killing. For the heart failure experiment, animals that developed heart failure were randomized to receive placebo or 3 MA for one month. 3 MA was administered intraperitoneally at a dose of $40 \mathrm{mg} / \mathrm{kg} /$ day to inhibit autophagy induction in vivo. Age-matched sham-operated animals were used as control ( $n=4$ in each group). The cross clamp surgery with gene transfer and $A A B$ was performed as previously described in detail. ${ }^{33,34}$ Briefly, the chest was opened at midline between the second and the fifth intercostals space. The aorta and the pulmonary arteries were cross-clamped simultaneously and the virus (Ad-GFP versus Ad-DN-JNK)/saline was injected into the LV. The cross clamp duration was $45 \mathrm{~s}$ and the adenovirus dose used was $200 \mathrm{PFU} / \mathrm{cell}$. After adenoviral delivery, $\mathrm{AAB}$ was performed and animals were studied 2 weeks later ( $n=4$ in each group).

Echocardiography. Transthoracic echocardiography was performed using a vivid 7 echocardiography apparatus with a $14 \mathrm{MHZ}$ probe (i13L probe, General Electric, New York, NY, USA). Long-axis parasternal views and short-axis parasternal two dimensional (2D) views, at the mid-papillary level, of the LV were obtained to calculate the LVEDV and LVESV volumes as well as the ejection fraction of the LV.

Invasive pressure-volume loop measurements of the LV. Hemodynamics were recorded subsequently through a Scisense P-V Control Unit (FY897B, London, ON, Canada). The intrathoracic inferior vena cava was transiently occluded to decrease venous return during the recording to obtain loadindependent $\mathrm{P}-\mathrm{V}$ relationships. Linear fits were obtained for end-systolic pressure volume relationships and EDPVR. At the end of the experiment, $50 \mu \mathrm{l}$ of $30 \% \mathrm{NaCl}$ were slowly injected into the external jugular vein for ventricular parallel conductance (Gp) measurement as previously described. ${ }^{35,36}$ Blood resistivity was measured using a special probe (Scisense). Volume measurements were initially obtained as blood conductance and calibrated using the Baan equation, ${ }^{37}$ and pressure sensors were calibrated as per manufacturer's instructions.

Statistical analysis. Results are shown as mean \pm S.D. Statistical significance was determined using Student-Newman-Keuls test. A $P$-value of $<0.05$ was considered statistically significant.

\section{Conflict of Interest}

The authors declare no conflict of interest.

Acknowledgements. This work was supported by NIH R01 HL083156, HL080498, HL093183 and P20HL100396 to (RJH) and by a 2011 Research Fellowship Award from the Heart Failure Society of America to (AHC).

1. Mann DL. Left ventricular size and shape: determinants of mechanical signal transduction pathways. Heart Fail Rev 2005; 10: 95-100.

2. Giorgi $C$, De Stefani D, Bononi A, Rizzuto R, Pinton P. Structural and functional link between the mitochondrial network and the endoplasmic reticulum. Int J Biochem Cell Biol 2009; 41: 1817-1827.

3. Walter L, Hajnoczky G. Mitochondria and endoplasmic reticulum: the lethal interorganelle cross-talk. J Bioenerg Biomembr 2005; 37: 191-206. 
4. Boncompagni S, Rossi AE, Micaroni M, Beznoussenko GV, Polishchuk RS, Dirksen RT et al. Mitochondria are linked to calcium stores in striated muscle by developmentally regulated tethering structures. Mol Biol Cell 2009; 20: 1058-1067.

5. Griffiths EJ, Rutter GA. Mitochondrial calcium as a key regulator of mitochondrial ATP production in mammalian cells. Biochim Biophys Acta 2009; 1787: 1324-1333.

6. Rizzuto R, Pozzan T. Microdomains of intracellular $\mathrm{Ca2+:}$ molecular determinants and functional consequences. Physiol Rev 2006; 86: 369-408.

7. Eisenberg-Lerner A, Bialik S, Simon HU, Kimchi A. Life and death partners: apoptosis, autophagy and the cross-talk between them. Cell Death Differ 2009; 16: 966-975.

8. Gross A, McDonnell JM, Korsmeyer SJ. BCL-2 family members and the mitochondria in apoptosis. Genes Dev 1999; 13: 1899-1911.

9. Zhang J, Ney PA. Role of BNIP3 and NIX in cell death, autophagy, and mitophagy. Cell Death Differ 2009; 16: 939-946.

10. Landes T, Emorine LJ, Courilleau D, Rojo M, Belenguer P, Arnaune-Pelloquin L. The BH3only Bnip3 binds to the dynamin Opa1 to promote mitochondrial fragmentation and apoptosis by distinct mechanisms. EMBO Rep 2010; 11: 459-465

11. Quinsay MN, Lee Y, Rikka S, Sayen MR, Molkentin JD, Gottlieb RA et al. Bnip3 mediates permeabilization of mitochondria and release of cytochrome $c$ via a novel mechanism. J Mol Cell Cardiol 2010; 48: 1146-1156.

12. Quinsay MN, Thomas RL, Lee $\mathrm{Y}$, Gustafsson AB. Bnip3-mediated mitochondrial autophagy is independent of the mitochondrial permeability transition pore. Autophagy 2010; 6: 17-24.

13. Glick D, Barth S, Macleod KF. Autophagy: cellular and molecular mechanisms. J Pathol 2010; 221: 3-12.

14. Mammucari C, Milan G, Romanello V, Masiero E, Rudolf R, Del Piccolo P et al. FoxO3 controls autophagy in skeletal muscle in vivo. Cell Metab 2007; 6: 458-471.

15. Metukuri MR, Beer-Stolz D, Namas RA, Dhupar R, Torres A, Loughran PA et al Expression and subcellular localization of BNIP3 in hypoxic hepatocytes and liver stress. Am J Physiol Gastrointest Liver Physiol 2009; 296: G499-G509.

16. Diwan A, Krenz M, Syed FM, Wansapura J, Ren X, Koesters AG et al. Inhibition of ischemic cardiomyocyte apoptosis through targeted ablation of Bnip3 restrains postinfarction remodeling in mice. J Clin Invest 2007; 117: 2825-2833.

17. Gang H, Hai Y, Dhingra R, Gordon JW, Yurkova N, Aviv Y et al. A novel hypoxia-inducible spliced variant of mitochondrial death gene bnip3 promotes survival of ventricular myocytes. Circ Res 2011; 108: 1084-1092.

18. Kubli DA, Quinsay MN, Huang $C$, Lee $Y$, Gustafsson AB. Bnip3 functions as mitochondrial sensor of oxidative stress during myocardial ischemia and reperfusion. Am J Physiol Heart Circ Physiol 2008; 295: H2025-H2031.

19. Regula KM, Ens K, Kirshenbaum LA. Inducible expression of BNIP3 provokes mitochondrial defects and hypoxia-mediated cell death of ventricular myocytes. Circ Res 2002; 91: 226-231.

20. Shao Z, Bhattacharya K, Hsich E, Park L, Walters B, Germann U et al. c-Jun N-terminal kinases mediate reactivation of Akt and cardiomyocyte survival after hypoxic injury in vitro and in vivo. Circ Res 2006; 98: 111-118.

21. Mammucari C, Schiaffino S, Sandri M. Downstream of Akt: FoxO3 and mTOR in the regulation of autophagy in skeletal muscle. Autophagy 2008; 4: 524-526.

22. Sandri M. Signaling in muscle atrophy and hypertrophy. Physiology (Bethesda) 2008; 23 160-170.
23. Greer EL, Brunet A. FOXO transcription factors at the interface between longevity and tumor suppression. Oncogene 2005; 24: 7410-7425.

24. Li HH, Kedar V, Zhang C, McDonough H, Arya R, Wang DZ et al. Atrogin-1/muscle atrophy F-box inhibits calcineurin-dependent cardiac hypertrophy by participating in an SCF ubiquitin ligase complex. J Clin Invest 2004; 114: 1058-1071.

25. Li HH, Willis MS, Lockyer P, Miller N, McDonough H, Glass DJ et al. Atrogin-1 inhibits Aktdependent cardiac hypertrophy in mice via ubiquitin-dependent coactivation of Forkhead proteins. J Clin Invest 2007; 117: 3211-3223.

26. Willis MS, Rojas M, Li L, Selzman CH, Tang RH, Stansfield WE et al. Muscle ring finger 1 mediates cardiac atrophy in vivo. Am J Physiol Heart Circ Physiol 2009; 296: H997-H1006.

27. Choukroun G, Hajjar R, Fry S, del Monte F, Haq S, Guerrero JL et al. Regulation of cardiac hypertrophy in vivo by the stress-activated protein kinases/c-Jun $\mathrm{NH}(2)$-terminal kinases. J Clin Invest 1999; 104: 391-398.

28. Choukroun G, Hajjar R, Kyriakis JM, Bonventre JV, Rosenzweig A, Force T. Role of the stress-activated protein kinases in endothelin-induced cardiomyocyte hypertrophy. J Clin Invest 1998; 102: 1311-1320.

29. Piper HM, Probst I, Schwartz P, Hutter FJ, Spieckermann PG. Culturing of calcium stable adult cardiac myocytes. J Mol Cell Cardiol 1982; 14: 397-412.

30. Wold LE, Ren J. Mechanical measurement of contractile function of isolated ventricular myocytes. Methods Mol Med 2007; 139: 263-270.

31. Kim M, Oh JK, Sakata S, Liang I, Park W, Hajjar RJ et al. Role of resistin in cardiac contractility and hypertrophy. J Mol Cell Cardiol 2008; 45: 270-280.

32. Del Monte F, Butler K, Boecker W, Gwathmey JK, Hajjar RJ. Novel technique of aortic banding followed by gene transfer during hypertrophy and heart failure. Physiol Genomics 2002; 9: 49-56.

33. Hajjar RJ, Schmidt U, Matsui T, Guerrero JL, Lee KH, Gwathmey JK et al. Modulation of ventricular function through gene transfer in vivo. Proc Natl Acad Sci USA 1998; 95: 5251-5256.

34. del Monte F, Hajjar RJ. Efficient viral gene transfer to rodent hearts in vivo. Methods Mol Biol 2003; 219: 179-193.

35. Pacher P, Nagayama T, Mukhopadhyay P, Batkai S, Kass DA. Measurement of cardiac function using pressure-volume conductance catheter technique in mice and rats. $\mathrm{Nat}$ Protoc 2008; 3: 1422-1434.

36. Porterfield JE, Kottam AT, Raghavan K, Escobedo D, Jenkins JT, Larson ER et al. Dynamic correction for parallel conductance, GP, and gain factor, alpha, in invasive murine left ventricular volume measurements. J Appl Physiol 2009; 107: 1693-1703.

37. Baan J, van der Velde ET, de Bruin HG, Smeenk GJ, Koops J, van Dijk AD et al. Continuous measurement of left ventricular volume in animals and humans by conductance catheter. Circulation 1984; 70: 812-823.

Cell Death and Disease is an open-access journal published by Nature Publishing Group. This work is licensed under the Creative Commons Attribution-Noncommercial-No Derivative Works 3.0 Unported License. To view a copy of this license, visit http://creativecommons.org/licenses/by-nc-nd/3.0/

\section{Supplementary Information accompanies the paper on Cell Death and Disease website (http://www.nature.com/cddis)}

